\title{
piRNA involvement in genome stability and human cancer
}

\author{
Miguel Moyano and Giovanni Stefani ${ }^{*}$
}

\begin{abstract}
PIWI-interacting RNAs (piRNAs) are a large family of small, single-stranded, non-coding RNAs present throughout the animal kingdom. They form complexes with several members of the PIWI clade of Argonaute proteins and carry out regulatory functions. Their best established biological role is the inhibition of transposon mobilization, which they enforce both at the transcriptional level, through regulation of heterochromatin formation, and by promoting transcript degradation. In this capacity, piRNAs and PIWI proteins are at the heart of the germline cells' efforts to preserve genome integrity. Additional regulatory roles of piRNAs and PIWI proteins in gene expression are becoming increasingly apparent.

PIWI proteins and piRNAs are often detected in human cancers deriving from germline cells as well as somatic tissues. Their detection in cancer correlates with poorer clinical outcomes, suggesting that they play a functional role in the biology of cancer. Nonetheless, the currently available information, while highly suggestive, is still not sufficient to entirely discriminate between a 'passenger' role for the ectopic expression of piRNAs and PIWI proteins in cancer from a 'driver' role in the pathogenesis of these diseases. In this article, we review some of the key available evidence for the role of piRNAs and PIWI in human cancer and discuss ways in which our understanding of their functions may be improved.
\end{abstract}

\section{Transposon mobilization and genome instability in cancer}

As most cancers stem from the accumulation of mutations, genome instability, defined as a propensity to have mutations, is an 'enabling characteristic' of tumor cells $[1,2]$. There are multiple sources of genome instability in cancer, ranging from exposure to environmental genotoxic substances, to endogenous generation of reactive species of a metabolic origin, resulting in DNA damage. In addition, the human genome carries a plethora of potential insertional mutagens in its own architecture, in the form of transposable elements (TEs) or 'jumping genes'. While sequences originating from TEs account for a staggering $45 \%$ of the entire human genome, only a relatively small set of $80-100$ transposable elements are still complete and capable of transposition [3].

The impact of TE mobilization on human cancer has only recently become measurable, thanks largely to progress in the field of high-throughput sequencing

\footnotetext{
*Correspondence: giovanni.stefani-1@unitn.it

Gene Expression Laboratory, Centre for Integrative Biology (CIBio), University of Trento, Trento 38123, Italy
}

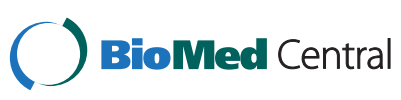

technologies. Evolution has endowed cells with a complex arsenal of counter-measures to keep potentially harmful mobilization of TEs in check. One such strategy is based on the action of specialized ribonucleoprotein (RNP) complexes, at the core of which lie members of the PIWI clade of Argonaute proteins and the small, noncoding piRNAs associated with them. While research in recent years has succeeded in unraveling several details of PIWI-interacting RNAs (piRNAs) biogenesis and function in transposon silencing, uncertainties remain on other aspects of their fascinating biology, such as the scope of their function in post-transcriptional regulation of gene expression and their suggested role in human cancer.

This article reviews the intersected fields of piRNA and transposon biology and discusses some of the still rather incomplete evidence in favor of a role of the PIWI/piRNA axis in human cancer.

\section{Retrotransposons and genome instability Transposable elements in the human genome}

The existence of transposable elements was first discovered in maize by Barbara McClintock in the 1940s and gained renewed attention about three decades later, 
when advancement in molecular biology made it possible to appreciate the universality of the phenomenon across living forms. Transposable elements of the human genome can be classified according to their mode of replication as 1) retrotransposons, which are transcribed into an RNA intermediate, and 2) DNA transposons, which do not need transcription to be mobilized (reviewed in [4]).

Much like retroviruses, retrotransposon transcripts need to be retrotranscribed into cDNA by a reverse transcriptase, which is itself encoded by the retrotransposon. In turn, retrotransposons are classified as 1) LTR retrotransposons, legacy of ancient germline retroviral infections and believed to be inactive in humans, 2) long interspersed elements 1 and 2 (LINE-1 and LINE- 2 or L1 and L2), and 3) short interspersed elements (SINEs), in turn belonging to the SINE-Alu and SVA classes. While LINEs encode a reverse transcriptase (and are therefore called 'autonomous'), SINEs do not and depend on the two proteins encoded by LINEs for their own replication and integration. DNA transposons constitute less than $2 \%$ of the human genome and depend on various transposases for their mobilization and insertion in their new position in the genome. Only a subset of about 80 to 100 copies of LINE-1 are competent for transposition in humans [5]. These L1s are therefore responsible for the entire retrotransposition activity still present in the human genome, as the two proteins they encode are hijacked by SINEs for their own cycle of retrotranscription and genomic integration.

LINE-1 elements are about $6 \mathrm{~kb}$ long and encode two proteins, called ORF1p and ORF2p. The 5'UTR carries an internal promoter, as well as an antisense promoter, whose function is not clear. With their astounding abundance of about 400,000 largely defective copies, they make up close to $17 \%$ of the human genome [3]. ORF1p is an RNA-binding protein required for the formation of the retrotransposon particle, while ORF2p carries the two crucial enzymatic activities required for retrotransposition: reverse transcription and endonuclease.

The LINE-1 transposition cycle begins with transcription, driven by the internal promoter, followed by export of the bicistronic mRNA to the cytoplasm, where translation of ORF1p and ORF2p takes place. The ORF1p protein shows a strong preference for binding the transcript molecule that encoded for itself, a phenomenon called cis-preference [6]. ORF1p's biochemical function is not entirely deciphered, but the co-crystal structure of ORF1p and LINE-1 RNA shows that trimers of the protein form a flexible nucleic acid chaperone, around which a single strand of RNA is wrapped, a spatial arrangement likely to contribute to the stability and nuclear import of the complex [7]. An L1 cytoplasmic RNP particle is thus formed, which also includes ORF2p, the second protein encoded by L1 elements.
While the full spectrum of ORF1p binding targets within the transcriptome has not been systematically studied, LINE-1 transcripts are not the only RNA bound by ORF1p: cellular transcripts present in the cytoplasm can be loaded in the complex, albeit at a much lower frequency, leading to the genomic insertion of their corresponding cDNAs in the form of pseudogenes [6]. Furthermore, transcripts from non-autonomous SINE (Alu, SVA) are frequently loaded into these RNP complexes, leading to their insertion into new genomic locations. Finally, the L1 RNP enters the nucleus, where the enzymatic activities of ORF2p lead to genomic integration.

ORF2p is a large protein, with reverse transcriptase and endonuclease activities. The widely accepted biochemical model of L1 integration, called target-primed reverse transcription (TPRT), postulates that, following a nick in one DNA strand corresponding to the recognition sequence $\left(5^{\prime}\right.$-TTAAA- $\left.3^{\prime}\right)$, the reverse transcriptase activity of ORF2p extends the DNA $3^{\prime}$ end using L1 RNA as its template [8]. The sequence specificity of the endonuclease is not the sole determinant of the choice of integration site. Recent in vitro studies show that the extent of base pairing within the DNA-RNA hybrid formed by the ten bases at the 3' end of LINE-1 RNA with the nicked DNA affects the efficiency of L1 ORF2pmediated DNA extension [9]. Such mechanistic studies are beginning to shed light on the complexity of the events leading to integration site choice, which results in a wide range of potential target sites [10].

The biochemical mechanism of L1 integration subsequent to retrotranscription initiation, including synthesis of the second strand of cDNA and ligation of the 3' ends of the newly retrotranscribed DNA into genomic DNA, is unknown. Such events result in some signature features surrounding LINE-1 integrated in the genome, such as target site duplication (TSD), stretches of identical 5-30 nucleotides at the two ends of the integrated L1 element [11]. The predominant occurrence of $5^{\prime}$ end deletion in integrated LINE-1 elements is most likely a consequence of the 3 ' to 5 ' direction of reverse transcription and makes the overwhelming majority of them incapable of retrotransposition.

\section{L1 retrotransposition: when and where}

Detection of abundant L1 transcripts and proteins brought forth the concept that transposition takes place actively in germ cells $[12,13]$. Subsequent studies in transgenic mice, while confirming the presence of L1 transcripts in the germline, failed to reveal frequent genomic insertion in this cell population; L1 elements appear instead to integrate in the genome in the early phases of embryonic development, mostly in somatic tissues, suggesting the presence of post-transcriptional mechanisms specifically devoted to the preservation of genomic integrity from TE insertion in gametes [14]. 
Despite the presence of multi-layered mechanisms of control in the germline (see below), transposons have obviously managed to trick these mechanisms at various times during evolution, becoming a major driving force in the shaping of genomes, from their prodigious expansion earlier in mammalian evolution to phylogenetically recent differences among primates [15-17]. On the other hand, the presence of L1 transcripts and non-heritable retrotransposition events in somatic cells indicates that TEs contribute to the establishment of mosaicism within an individual [14]. There is abundant evidence of somatic retrotransposition in somatic cell types such as testicular endothelium and Leydig cells, myocardium, and various types of primary human cells in culture [18-21]. Somatic retrotransposition in the brain has been extensively documented, in species ranging from Drosophila to human [22] (reviewed in [23]).

In all, the mounting evidence of retrotransposition in somatic tissue, in particular in the brain, challenges the concept that all cells in an individual's soma have identical genomes (barring the genomic rearrangements in lymphocytes), and supports a scenario where each individual's soma is a mosaic of somewhat genomically different cell populations. In this perspective, mobilization of TEs can be seen as beneficial, providing a molecular mechanism contributing to the astounding variety of neuron types and connectivity, for instance [23]. On the other hand, the presence of an active insertional mutagen within the genome can also be a powerful source of instability, leading to a variety of human diseases.

\section{Mechanisms of retrotransposon-induced genome instability}

The mechanisms through which TE mobilization can lead to potentially harmful mutations are manifold $[4,24]$. TEs (mostly Alu elements) provide a vast repertoire of homologous sequences scattered throughout the genome that can be involved in non-allelic homologous recombination (NAHR), which leads to deletions and duplications $[25,26]$. NAHR-induced mutations are estimated to be vastly more frequent than all the other TEinduced mutations in human cancers (reviewed in [27]). In addition, integration of TEs in exons can cause disease by creating frameshifts, leading to premature stop codons and nonsense-mediated decay, or by inducing exon skipping. Furthermore, TE, in particular Alu, can introduce additional splicing sites upon incorporation into exons ('exonization'), creating novel, potentially detrimental alternative splicing isoforms [28]. Less frequently, insertions of TEs can also cause large deletions of coding sequence. The relatively weak polyadenylation sites of LINEs are sometimes not processed as such, leading to read-through transcription of sequences belonging to a flanking gene, their incorporation in an active element, and their transduction to a novel genomic location [29].

Additionally, the presence of many polyadenylation sites in the sense strand of LINE-1, while likely to reduce mobilization of functional elements, contributes to worsen the impact of their insertion, by imparting sites of transcriptional pausing and decreasing the overall efficiency of transcription of the host gene [30]. LINE-1 can also disrupt gene expression at the transcriptional level by inducing the antisense transcription of genes flanking its integration site through antisense promoter activity present in its 5'UTR. Additional mechanisms of alteration of gene expression, which might cause human disease, include silencing of flanking genes by heterochromatization of the transposable element as a result of the cell's control mechanisms and other less frequent molecular phenomena (reviewed in [24]).

The range of human pathologies associated with documented insertion of retrotransposons is vast and expanding. Hancks and Kazazian compiled a list of 96 instances of single-gene disease described in the literature, from hemophilia to diabetes to cancer $[4,23,31,32]$.

\section{Mobilization of transposons and cancer}

As mutagens with very limited sequence requirements for insertion, LINE and SINE are natural candidate pathogenic factors in cancer, a disease caused by mutations. In addition, exposure to some environmental factors that are known to increase cancer risk also promotes TE transcription and promoter demethylation, supporting the notion that transposition might be a causal mechanism of neoplasm [33-35]. Aging, a major risk factor for cancer in humans, is also accompanied by increased TE mobilization in yeast and, more broadly, increased somatic mosaicism in humans [36-38].

Direct evidence of transposon activation in human cancer and transformed cell lines have been gathered over the past 20 years. Components of TEs, both transcripts and proteins, are elevated in cancer compared to normal tissues [33,39-43]. Consistent with a higher level of transposon expression, methylation of transposon promoters is decreased in cancer [44-47] (reviewed in [48]). Both phenomena correlate with worse prognosis and higher levels of metastasis, substantiating a pathogenic role for transposon mobilization [43-47] (reviewed in [48]). These observed correlations grant plausibility to a model in which prolonged exposure to adverse environmental factors in aging organisms leads to increased retrotransposition, with, in turn, increased risk of abrogation of tumor suppressor activities by one of the several mechanisms outlined above.

As it is often the case for cancer mutations, an outstanding question in the field has been whether these transposon activities are 'driver' phenomena or consequence of 
the general derangement of gene expression and genome instability that are hallmarks of cancer. Disruption of genes with a well-established tumor suppressor activity by retrotransposon integration provides a strong correlative evidence for a pathogenetic role for such mutations. A first instance of such occurrence was detected in 1992 in a survey by Southern blot of the APC tumor suppressor gene in 150 colorectal cancer samples. The shift in size of one fragment by several kilobases led to the realization that a LINE-1 sequence was inserted in the gene [49].

Developments in high-throughput sequencing technologies are beginning to allow a full-genome outlook on the impact of TEs on carcinogenesis that was not available just a few years ago. In a recent such study, a survey of 43 full-genome paired-end sequencing databases from five different cancer types revealed 194 instances of novel, cancer-specific somatic insertions [50]. The pool of 64 loci that were affected by these insertions included genes associated with tumor suppressor and cell adhesion functions, with an overall enrichment of frequently mutated genes, a feature associated with a driver role in tumorigenesis [51]. The expression levels of the genes targeted by transposition were decreased, consistent with a functional role of transposon insertion [50].

In disagreement with the conclusions of this report, the analysis of a large dataset of 290 cancer genomes, while identifying a large number $(2,850)$ of new somatic transposition events occurring in cancer, failed to identify insertions with obvious pathogenic implications, such as disruption of tumor suppressor genes, nor did it detect changes in gene expression of the genes targeted by TE integration [52]. Using a method called retrotransposon capture sequencing (RC-seq), 19 hepatocellular carcinoma (HPCC) genomes were analyzed for novel germline and somatic transposition [53]. Two likely L1-mediated tumorigenic mechanisms were unveiled. In $20 \%$ of the analyzed genomes, the expression of the tumor suppressor mutated in colorectal cancer (MCC) was drastically reduced by germline L1 insertions, resulting in activation of the oncogenic $\mathrm{Wnt} / \beta$-catenin pathway. Furthermore, somatic insertion in an intronic transcriptional enhancer resulted in the interruption of a self-repression loop, leading to increased expression of the oncogene ST18 [53].

In summary, while the overwhelming majority of retrotransposon activity in tumors is not likely to be directly driving neoplastic transformation, active retrotransposition is a generator of genomic variability, from which occasionally mutations that impair tumor suppressor genes can arise [52,53].

\section{Guardians of genome integrity: PIWIs and piRNAs PIWI and piRNA biogenesis and mechanism of action}

Several layers of control stem mobilization and spreading of transposons in normal cells. Different stages of a retrotransposon's life cycle are targeted by such control mechanisms: the exonuclease Trex1, which is also involved in cell response to retroviral infection, degrades single-stranded cDNA deriving from retrotransposons, while the APOBEC3 cytidine deaminase family inhibits retrotransposition through a variety of molecular processes [54] (reviewed in [24]). In addition to these mechanisms, studies during the last decade uncovered how the cell leverages a complex level of RNA-based control strategies to inhibit mobile elements at the early stages of transcription as well as post-transcriptionally.

The functional architecture of the PIWI/piRNAs axis shows similarities with other two well-characterized small, non-coding RNA-based regulatory pathways, miRNAs and siRNAs, chiefly among them the role of the RNA component as a specificity factor through base pairing, in the context of a RNP effector complex that includes members of the AGO family of proteins. Biogenesis sets piRNAs apart from the other families of small, non-coding RNAs: while double-stranded or stem-loop precursors of siRNAs and miRNAs are processed by the RNAse III Dicer, piRNAs are mostly transcribed as large (up to $200 \mathrm{~kb}$ ) single-stranded precursors, which are processed independently from Dicer. In addition, piRNAs form functional complexes exclusively with members of the PIWI clade of Argonaute proteins.

The piRNAs of Drosophila and mice can be grouped in three classes according to their origin: 1) repeatassociated piRNAs derived from intergenic loci, called piRNA clusters, that are enriched in transposon fragments in Drosophila, zebrafish, and in a subset of piRNA clusters in mice; 2) mRNA-derived piRNAs derived from the 3'UTR of mRNAs; and 3) long, non-coding RNAderived piRNAs [55]. The biogenesis and functions of repeat-associated piRNAs are much better understood than the other two classes (reviewed in $[55,56]$ ).

In the simplest scenario, Drosophila gonad somatic cells, the primary transcript is first cleaved, probably by the riboendonuclease Zucchini. The 3' fragment is incorporated in PIWI proteins and trimmed to a final length of $\sim 25$ nt by a $3^{\prime}$ to $5^{\prime}$ exonuclease. The $5^{\prime}$ end residue of the piRNA incorporated in PIWI shows a strong bias for uridine residues, while the 2 ' hydroxy group at the 3 ' end is methylated by the enzyme Hen1. The final length of piRNAs is likely dictated by the extent of protection from exonuclease trimming, as each PIWI protein binds a subpopulation of piRNAs with slightly different modal sizes (25.7, 24.7, and $24.1 \mathrm{nt}$ for Piwi, Aubergine (Aub), and AGO3, respectively) [57]. The PIWI/piRNA complex enters the nucleus, is targeted to actively transcribed nascent transposon by recognition of sequences complementary to its piRNA component, and inhibits further transcription by recruiting histone methyltransferases, which will lead to the establishment of transcriptionally silent heterochromatin [58]. 
In Drosophila germ cells, this piRNA-mediated inhibition of transposon mobilization is amplified through the 'ping-pong' mechanism (Figure 1). Primary piRNAs, antisense to transposons, are incorporated in the Aub PIWI protein and target complementary transposon RNA sequences in the cytoplasm. Aub cleaves transposon RNA between residues complementary to the 10th and 11th piRNA residues. The resulting 3' fragment of the transposon RNA is incorporated in AGO3, another PIWI protein, trimmed and modified with a 2' O-methylation, thus forming a secondary piRNA, which will therefore begin with ten residues complementary to the first ten residues of the primary piRNA, with an adenosine as its tenth residue complementary to the first uridine residue of the primary piRNA. When the AGO3-secondary piRNA complex targets antisense strand piRNA cluster transcripts, it will produce new piRNAs identical to primary piRNAs, which will be incorporated in Aubergine complexes, perpetuating the cycle. This mechanism degrades transposon RNA while amplifying the pool of piRNA/PIWI complexes that can inhibit its expression at the transcriptional and post-transcriptional levels (reviewed in [55]).

In mammals, piRNA/PIWI mechanisms display even more variety. The earliest piRNA population, bound to MILI and MIWI2, inhibits transcription of transposons independently of cleavage activity and fades away soon after birth [59]. A mechanism of amplification closely resembling Drosophila ping-pong is carried out in prenatal murine testis by the PIWI protein MILI and provides secondary piRNAs bound by MIWI2 [60]. Post-natally, two populations of piRNAs are found in pre-pachytene and pachytene spermatocytes, respectively. The former derives from 3'UTR regions of mRNAs, while the latter, an extremely abundant population of short RNAs, stems from lncRNAs. While genetic ablation experiments show that both these piRNA classes are required for correct spermatogenesis, their molecular function remains a mystery. These very abundant piRNAs do not bear any complementarity to transposable elements, suggesting that they might have a different molecular target. A recent report suggests that pachytene piRNAs and MIWI target up to $40 \%$ of mRNAs in elongating spermatids, leading to their deadenylation and degradation during spermiogenesis [61].

Further evidence of the wide scope of the PIWI/ piRNA-mediated regulation of gene expression comes from studies in simpler organisms. In Caenorhabditis elegans, piRNA-like molecules, called 21U RNAs, are at the center of a complex sequence-scanning system that enables cells to detect non-self sequences such as mobile

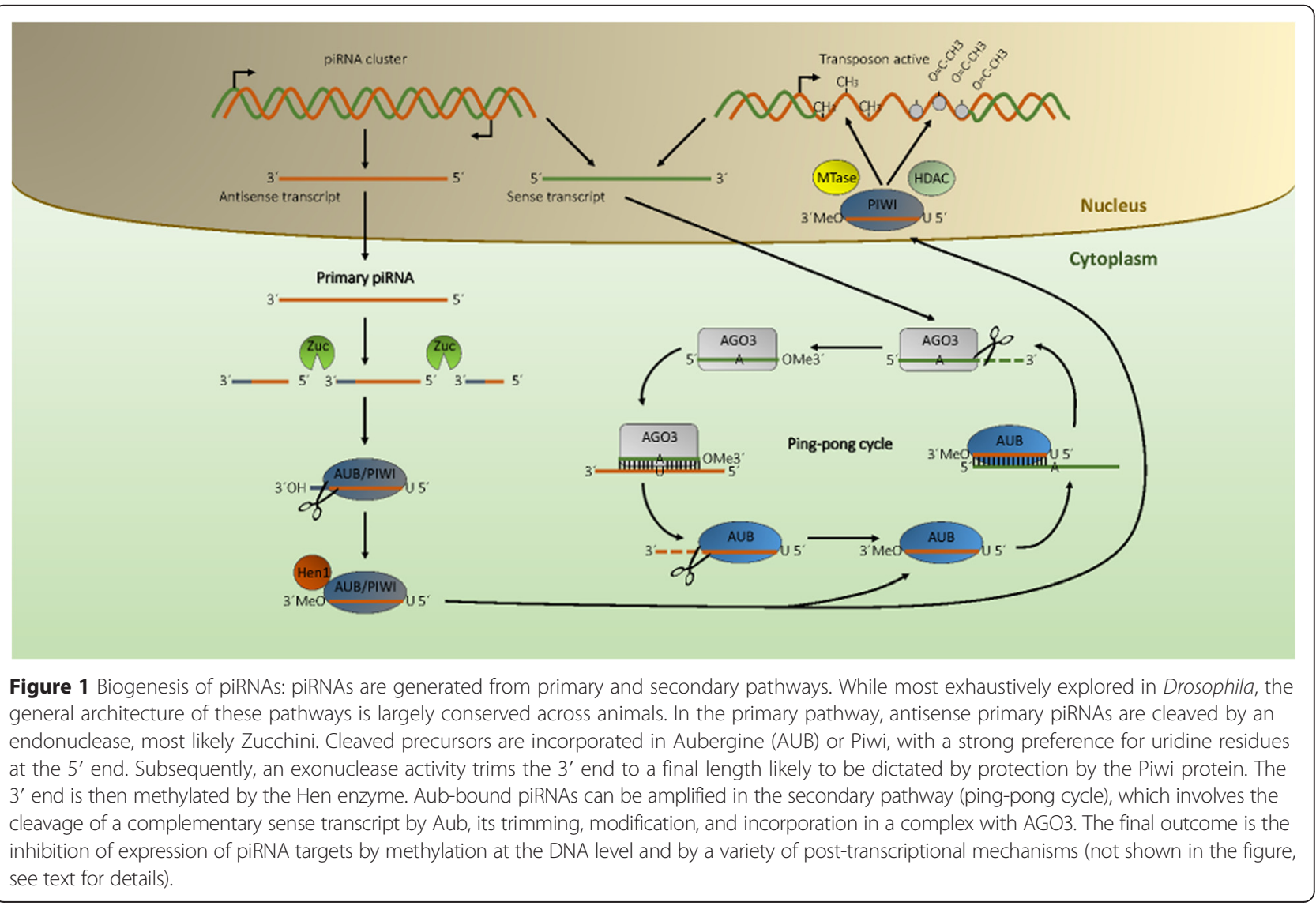


elements or, occasionally, transgenes and suppresses their expression. This system relies on the production of a large population of secondary siRNAs: upon recognition of a non-self mRNA by $21 \mathrm{U}$ RNAs, in a complex with the PIWI protein PRG-1, the so-called 22G RNAs are produced by an RNA-dependent RNA polymerase, using the foreign RNA as template [61-64]. These secondary siRNAs target an effector Argonaute protein, called WAGO-9, to complementary sequences (probably nascent transcripts) in the nucleus, where heterochromatin proteins, DNA methylases, and other factors are recruited to the site of transcription through interactions with WAGO-9, contributing to silencing of the non-self sequences. An opposing scanning system exists, which guarantees the expression of 'self' mRNAs. The sequence diversity of $21 \mathrm{U}$ RNAs is such that they could conceivably target virtually the entire transcriptome, similarly to mammalian 'pachytene' piRNAs: neutralizing their inhibitory effect, yet another group of Argonaute proteins (CSR-1, ALG-3, and ALG-4) forming complex with a separate group of $22 \mathrm{G}$ and $26 \mathrm{G}$ RNAs promote the proper expression of the genes required for germline development and maintenance [65]. Amazingly, such positive regulators of small RNA/Argonaute complexes are packed in the sperm and transmitted to the zygote, thus providing a vehicle for trans-generational transmission of epigenetic information [65] (reviewed in [66]). Ciliated protozoa also show evidence of genomescanning mechanisms based on small RNAs that share piRNA features. These scanning RNAs (scnRNAs) regulate programmed DNA elimination, the process by which extensive tracts of DNA are removed from the genome of the haploid micronuclei when they conjugate to form 'somatic' macronuclei. In Tetrahymena and Paramecium, scRNAs mark the DNA sequences that are designated for elimination, while in Oxytricha trifallax, piRNAs mark genomic sequences to be retained $[67,68]$.

In addition to chromatin silencing, piRNAs control transposon and mRNA expression in the cytoplasm through degradation and possibly through translational inhibition and intracellular localization (reviewed in [69]). Close to one third of human mRNAs carry transposon-derived sequences in their 3'UTR, making them potential targets for PIWI/piRNA complexes [70]. During maternal nanos mRNA elimination in Drosophila early development, piRNAs complementary to regions of the 3'UTR and components of the piRNA pathway are required for its deadenylation and degradation [71]. The ability of transposon-derived sequences to induce instability to reporters has also been shown in mammalian systems [72]. These evidence of the ability of the piRNA surveillance system to degrade mRNAs and the pervasive diffusion of transposon sequences in the human genome should be taken into account when considering the possible role of ectopically expressed PIWI proteins and piRNAs in human cancer.

\section{PIWI, piRNAs, and cancer}

PIWI was originally discovered as a factor required for the maintenance of germline stem cells [73]. Members of the PIWI clade Argonaute are expressed in several stem cell populations across organisms and tissues, most robustly in the male germline in adult mammals (reviewed in [74]). Additionally, PIWIs are expressed in a large number of human cancers, of both germline and somatic origin, such as seminomas, multiple myeloma, and prostate, hepatocellular, breast, gastrointestinal, ovarian, and endometrial cancer, among others, as well as murine breast tumors, rhabdomyosarcoma, and medulloblastoma [75-78].

As it is often the case, the question arises of whether such findings point to an active role of PIWI in carcinogenesis, as opposed to representing a mere byproduct of dysregulated gene expression in cancer. Even though the field suffers from a dearth of functional studies, fairly solid correlation between expression of PIWI in cancer and a record of poorer clinical outcome suggest an impact on the biology of tumors. The expression of PIWI proteins in gastric, colorectal, breast, and cervical cancer, soft tissue sarcoma, adenocarcinoma of the pancreas, hepatocellular carcinoma, glioma, and esophageal squamous cell carcinoma correlates with a significant worse prognosis [78-86] (reviewed in [87]).

The mechanisms through which ectopic expression of PIWI affects the clinical outcome of cancers are largely unexplored. In one case, expression of PIWIL2 in three different cell types has been shown to profoundly affect the transcriptome, leading to a marked increase of the antiapoptotic gene $\mathrm{Bcl}-\mathrm{X}_{\mathrm{L}}$, as well as Stat3 and cyclin D1. These changes in gene expression were accompanied by reduced apoptosis, increased proliferation, and transformation [78]. In contrast, the simultaneous ectopic expression of piRNAs with PIWI proteins has been only documented in a handful of cases. In gastric cancer and multiple myeloma cell lines, piRNA-823 levels affect tumor aggressiveness, albeit, strangely, in opposite directions: in gastric cancer cells, piRNA-823 has an overall tumor suppressor activity, while in multiple myeloma, it promotes cancer development, suggesting perhaps functional interactions with cell-specific factors $[77,88]$.

Overall, the current picture of the role of the piRNA/ PIWI axis in human cancer is probably incomplete, mirroring the gaps in our grasp of its physiological role. If the sole function of piRNAs was to inhibit the mobilization of transposons, one would expect the ectopic expression of PIWI proteins in cancer to exert an overall antagonistic effect to tumor development and progression. This is not the case in the overwhelming 
majority of instances in which levels of PIWI have been perturbed in tumor cells: ectopic expression of PIWI actually imparts a more aggressive cancer behavior, and its inhibition reverses such phenotype. One can envision a scenario in which widespread demethylation leads to both activation of transposons and expression of PIWI proteins, or, alternatively, one in which the expression of PIWI proteins is somehow reactive to the activation of transposons through some unknown mechanism [89,90]. Either way, once expressed in the wrong cell types and at the wrong developmental time, PIWI proteins are likely to be engaged not exclusively in the repression of transposon mobilization but also in repressive interactions with RNAs they are not exposed to in germline cells. Post-transcriptional inhibition of gene expression by piRNAs through degradation or translational inhibition could be responsible for the documented tumorpromoting effects. In such a scenario, PIWI/piRNA procancer action would be reminiscent of the oncogenic role of some miRNA-Ago complexes such as miR-155, miR-21, or the miR-17-92 cluster [91-93]. The two modes of action of PIWI/piRNAs, transposon inhibition and post-transcriptional silencing of mRNAs, could still mechanistically be two sides of the same coin, as many mRNAs include extensive transposon-derived sequences [70]. Additionally, it cannot be ruled out at present that PIWI's and possibly piRNAs' role in cancer could be completely decoupled from control of transposon mobilization. As mentioned in the previous section, PIWI, piRNAs, and the Argonaute proteins WAGO-9 and CSR-1 and their cognate 22G and 26G RNAs are intertwined in a complex system that allows C. elegans to differentiate between 'self' from 'non-self' RNA [65]. Are mammalian PIWIs and piRNAs also part of an as yet undiscovered mammalian mechanism of vigilance on the proper expression of 'self' RNA, and is the derangement of such system somewhat at play in cancer? While vertebrates seem to lack 22G and 26G RNAs, the incredibly high number and sequence diversity of the 'pachytene' piRNAs associated to MIWI in mouse provides a repertoire of small, non-coding RNA that could, in principle, target the entire human transcriptome through imperfect complementarity. It is tempting to speculate that the improper activation of such a far-reaching apparatus normally devoted to the monitoring of proper gene expression in the germline may have profound consequence for the biological behavior of cancer.

What are the broader implications of PIWI and possibly piRNA expression in cancer cells? One suggestion comes from a Drosophila model of brain cancer: the ablation of the l(3)mbt gene, a member of the polycomb group (PcG) of proteins, leads to the development of brain tumors. Expression profiling revealed a 'l(3)mbt tumor signature' of 102 upregulated genes, which included piwi. Mutation of piwi was sufficient to suppress l(3)mbt malignant growth, providing strong in vivo evidence for its requirement for $l(3) m b t$ tumor formation. Remarkably, 26 out of 102 upregulated genes that constitute the 'l(3)mbt tumor signature' are required for germline development and maintenance. They include vasa and aubergine, which are, like piwi, involved in the piRNA pathway and were able to suppress the l(3)mbt penotype [94]. An extraordinary coordinated ectopic expression of genes normally restricted to the germline has also been observed in the C. elegans 'soma to germline' switch upon suppression of lin-35, an ortholog of RB (retinoblastoma protein), which interacts functionally and associates physically with $l(3) m b t$, as well as in longlived mutants of the insulin-like pathway $[95,96]$. Additionally, long-running efforts in the medical community to identify cancer-specific antigen for immunotherapy have resulted in the identification of tens of genes whose expression is exquisitely restricted to the male germline but becomes ectopically activated in a coordinated fashion in a variety of tumors [97]. The products of these genes have been therefore called cancer/testis (CT) antigens. The common thread of the ectopic, coordinated expression of genes that are normally germline-restricted in somatic cancer has led to the speculation that, by conferring biological features typical of the germline, CT antigens might contribute to the biology of cancer cells [97]. Cellular behaviors observed in gametogenesis, such as immortalization, implantation, and migration, may be seen as corresponding to, respectively, transformation, invasion, and metastasis in the context of cancer, while other phenomena such as global hypomethylation, active angiogenesis, and immune evasion are common to both cell types [97]. In this scenario, PIWI proteins ectopically expressed in tumors could contribute to confer features of actively replicating germline stem cells to cancer stem cells.

\section{Conclusions and perspectives}

Seventeen years after the discovery of PIWI and eight after that of piRNAs, the extent of their role in cancer, suggested by ectopic expression and correlation to clinical outcome, is still far from being clearly understood. Addressing the following questions would go a long way in substantially advancing this area of cancer biology:

1) Are piRNAs ectopically expressed in cancer? While PIWI protein expression in cancer is quite extensively documented, piRNAs have been detected in a handful of cases. These include a Drosophila model of brain tumor and human multiple myeloma and gastric cancer samples $[77,88,94]$. The biogenesis of piRNAs is a complex process that 
needs numerous factors, the expression of many of which has not been studied in most cancers where PIWI is expressed. In the absence of piRNAs, PIWI could perhaps bind other RNAs or engage in functional interactions of a radically different kind.

2) Which genes are regulated by piRNAs and PIWI proteins in cancer? The understanding of the biological functions of any trans-regulator of gene expression is greatly advanced by the identification of its functional targets. The field of RNA-binding protein and miRNA-Argonaute biology has benefited from addressing this question through experimental approaches such as high-throughput sequencing of RNA isolated by crosslinking immunoprecipitation (HITS-CLIP) or crosslinking, ligation, and sequencing of hybrids (CLASH) $[98,99]$. HITS-CLIP for Mili and Miwi in mouse testis reached the unexpected conclusion that Miwi binds mRNAs in the absence of a piRNA guide [100]. Can this finding be confirmed in cancer? Does the identity of genes regulated by ectopically expressed PIWI proteins point to a specific cellular process?

3) Is the ectopic expression of PIWI proteins and piRNAs driving cancer development and progression? More extensive studies in vertebrate animal models are necessary to address this fundamental question. In Drosophila, the requirement of piwi for brain tumor formation has been elegantly demonstrated in l(3)mbt, piwi double mutants. Establishing similar models of ectopic expression of PIWI and piRNAs in mammalian model organisms will provide necessary information on their capability to exert a causative role in the initiation and progression of neoplasms.

\section{Competing interests}

The authors declare that they have no competing interests.

\section{Authors' contributions}

MM and GS drafted the manuscript. Both authors read and approved the final manuscript.

Received: 19 November 2014 Accepted: 31 March 2015

Published online: 21 April 2015

\section{References}

1. Hanahan D, Weinberg RA. The hallmarks of cancer. Cell. 2000;100:57-70.

2. Hanahan D, Weinberg RA. Hallmarks of cancer: the next generation. Cell. 2011;144:646-74

3. Lander ES, Linton LM, Birren B, Nusbaum C, Zody MC, Baldwin J, et al. Initial sequencing and analysis of the human genome. Nature. 2001;409:860-921.

4. Hancks DC, Kazazian Jr HH. Active human retrotransposons: variation and disease. Curr Opin Genet Dev. 2012;22:191-203 [Molecular and Genetic Bases of Disease].

5. Brouha B, Schustak J, Badge RM, Lutz-Prigge S, Farley AH, Moran JV, et al Hot L1s account for the bulk of retrotransposition in the human population. Proc Natl Acad Sci U S A. 2003;100:5280-5.

6. Wei W, Gilbert N, Ooi SL, Lawler JF, Ostertag EM, Kazazian HH, et al. Human L1 retrotransposition: cis preference versus trans complementation. Mol Cell Biol. 2001;21:1429-39.
7. Khazina E, Truffault V, Büttner R, Schmidt S, Coles M, Weichenrieder O Trimeric structure and flexibility of the L1ORF1 protein in human L1 retrotransposition. Nat Struct Mol Biol. 2011;18:1006-14.

8. Luan DD, Korman MH, Jakubczak JL, Eickbush TH. Reverse transcription of R2Bm RNA is primed by a nick at the chromosomal target site: a mechanism for non-LTR retrotransposition. Cell. 1993;72:595-605.

9. Monot C, Kuciak M, Viollet S, Mir AA, Gabus C, Darlix J-L, et al. The specificity and flexibility of $L 1$ reverse transcription priming at imperfect T-tracts. PLoS Genet. 2013;9, e1003499.

10. Viollet S, Monot C, Cristofari G. L1 retrotransposition. Mob Genet Elem. 2014;4, e28907.

11. Ostertag EM, Kazazian Jr HH. Biology of mammalian L1 retrotransposons. Annu Rev Genet. 2001;35:501-38.

12. Branciforte D, Martin SL. Developmental and cell type specificity of LINE-1 expression in mouse testis: implications for transposition. Mol Cell Biol. 1994;14:2584-92.

13. Trelogan SA, Martin SL. Tightly regulated, developmentally specific expression of the first open reading frame from LINE-1 during mouse embryogenesis. Proc Natl Acad Sci. 1995;92:1520-4.

14. Kano H, Godoy I, Courtney C, Vetter MR, Gerton GL, Ostertag EM, et al. L1 retrotransposition occurs mainly in embryogenesis and creates somatic mosaicism. Genes Dev. 2009;23:1303-12.

15. Zhou Y-H, Zheng JB, Gu X, Saunders GF, Yung W-KA. Novel PAX6 binding sites in the human genome and the role of repetitive elements in the evolution of gene regulation. Genome Res. 2002;12:1716-22.

16. Hormozdiari F, Konkel MK, Prado-Martinez J, Chiatante G, Herraez IH, Walker JA, et al. Rates and patterns of great ape retrotransposition. Proc Natl Acad Sci. 2013;110:13457-62

17. Marchetto MCN, Narvaiza I, Denli AM, Benner C, Lazzarini TA, Nathanson JL, et al. Differential L1 regulation in pluripotent stem cells of humans and apes. Nature. 2013;503:525-9.

18. Kubo S, Seleme Mdel C, Soifer HS, Perez JLG, Moran JV, Kazazian HH, et al. $\mathrm{L} 1$ retrotransposition in nondividing and primary human somatic cells. Proc Natl Acad Sci. 2006;103:8036-41.

19. Shi X, Seluanov A, Gorbunova V. Cell divisions are required for $L 1$ retrotransposition. Mol Cell Biol. 2007;27:1264-70.

20. Lucchinetti E, Feng J, da Silva R, Tolstonog GV, Schaub MC, Schumann GG, et al. Inhibition of LINE-1 expression in the heart decreases ischemic damage by activation of Akt/PKB signaling. Physiol Genomics. 2006;25:314-24.

21. Ergün S, Buschmann C, Heukeshoven J, Dammann K, Schnieders F, Lauke H, et al. Cell type-specific expression of LINE-1 open reading frames 1 and 2 in fetal and adult human tissues. J Biol Chem. 2004;279:27753-63.

22. Muotri AR, Chu VT, Marchetto MCN, Deng W, Moran JV, Gage FH. Somatic mosaicism in neuronal precursor cells mediated by $L 1$ retrotransposition. Nature. 2005;435:903-10.

23. Erwin JA, Marchetto MC, Gage FH. Mobile DNA elements in the generation of diversity and complexity in the brain. Nat Rev Neurosci. 2014;15:497-506.

24. Goodier JL, Kazazian Jr HH. Retrotransposons revisited: the restraint and rehabilitation of parasites. Cell. 2008;135:23-35.

25. Han K, Lee J, Meyer TJ, Remedios P, Goodwin L, Batzer MA. L1 recombination-associated deletions generate human genomic variation. Proc Natl Acad Sci. 2008;105:19366-71.

26. Sen SK, Han K, Wang J, Lee J, Wang H, Callinan PA, et al. Human genomic deletions mediated by recombination between Alu elements. Am J Hum Genet. 2006;79:41-53.

27. Belancio VP, Roy-Engel AM, Deininger PL. All y'all need to know 'bout retroelements in cancer. Semin Cancer Biol. 2010;20:200-10 [Repetitive Elements and Genome Instability].

28. Ponicsan SL, Kugel JF, Goodrich JA. Genomic gems: SINE RNAs regulate mRNA production. Curr Opin Genet Dev. 2010;20:149-55 [Chromosomes and Expression Mechanisms].

29. Holmes SE, Dombroski BA, Krebs CM, Boehm CD, Kazazian HH. A new retrotransposable human L1 element from the LRE2 locus on chromosome 1q produces a chimaeric insertion. Nat Genet. 1994;7:143-8.

30. Perepelitsa-Belancio V, Deininger P. RNA truncation by premature polyadenylation attenuates human mobile element activity. Nat Genet. 2003;35:363-6.

31. Kazazian HH, Wong C, Youssoufian H, Scott AF, Phillips DG, Antonarakis SE. Haemophilia A resulting from de novo insertion of $L 1$ sequences represents a novel mechanism for mutation in man. Nature. 1988;332:164-6. 
32. Kaer K, Speek M. Retroelements in human disease. Gene. 2013;518:231-41.

33. Giorgi G, Marcantonio P, Re BD. LINE-1 retrotransposition in human neuroblastoma cells is affected by oxidative stress. Cell Tissue Res. 2011;346:383-91.

34. Stribinskis V, Ramos KS. Activation of human long interspersed nuclear element 1 retrotransposition by benzo(a)pyrene, an ubiquitous environmental carcinogen. Cancer Res. 2006:66:2616-20.

35. El-Sawy M, Kale SP, Dugan C, Nguyen TQ, Belancio V, Bruch H, et al. Nickel stimulates $L 1$ retrotransposition by a post-transcriptional mechanism. J Mo Biol. 2005;354:246-57.

36. Maxwell PH, Burhans WC, Curcio MJ. Retrotransposition is associated with genome instability during chronological aging. Proc Natl Acad Sci. 2011;108:20376-81.

37. Laurie CC, Laurie CA, Rice K, Doheny KF, Zelnick LR, McHugh CP, et al. Detectable clonal mosaicism from birth to old age and its relationship to cancer. Nat Genet. 2012;44:642-50.

38. Jacobs KB, Yeager M, Zhou W, Wacholder S, Wang Z, Rodriguez-Santiago B, et al. Detectable clonal mosaicism and its relationship to aging and cancer. Nat Genet. 2012;44:651-8.

39. Iskow RC, McCabe MT, Mills RE, Torene S, Pittard WS, Neuwald AF, et al. Natural mutagenesis of human genomes by endogenous retrotransposons. Cell. 2010;141:1253-61.

40. Baba Y, Huttenhower C, Nosho K, Tanaka N, Shima K, Hazra A, et al. Epigenomic diversity of colorectal cancer indicated by LINE-1 methylation in a database of 869 tumors. Mol Cancer. 2010;9:125.

41. Belancio VP, Roy-Engel AM, Pochampally RR, Deininger P. Somatic expression of LINE-1 elements in human tissues. Nucleic Acids Res. 2010;38:3909-22.

42. Solyom S, Ewing AD, Rahrmann EP, Doucet $T$, Nelson HH, Burns MB, et al. Extensive somatic L1 retrotransposition in colorectal tumors. Genome Res. 2012;22:2328-38.

43. Chen L, Dahlstrom JE, Chandra A, Board P, Rangasamy D. Prognostic value of LINE-1 retrotransposon expression and its subcellular localization in breas cancer. Breast Cancer Res Treat. 2012;136:129-42.

44. Ogino S, Nosho K, Kirkner GJ, Kawasaki T, Chan AT, Schernhammer ES, et al. A cohort study of tumoral LINE-1 hypomethylation and prognosis in colon cancer. J Natl Cancer Inst. 2008;100:1734-8.

45. Pattamadilok J, Huapai N, Rattanatanyong P, Vasurattana A, Triratanachat S, Tresukosol D, et al. LINE-1 hypomethylation level as a potential prognostic factor for epithelial ovarian cancer. J Gynecol Cancer. 2008;18:711-7.

46. Shuangshoti S, Hourpai N, Pumsuk U, Mutirangura A. Line-1 hypomethylation in multistage carcinogenesis of the uterine cervix. Asian Pac J Cancer Prev APJCP. 2007:8:307-9.

47. Yegnasubramanian S, Haffner MC, Zhang Y, Gurel B, Cornish TC, Wu Z, et al. DNA hypomethylation arises later in prostate cancer progression than CpG island hypermethylation and contributes to metastatic tumor heterogeneity. Cancer Res. 2008;68:8954-67.

48. Carreira PE, Richardson SR, Faulkner GJ. L1 retrotransposons, cancer stem cells and oncogenesis. FEBS J. 2014;281:63-73.

49. Miki Y, Nishisho I, Horii A, Miyoshi Y, Utsunomiya J, Kinzler KW, et al. Disruption of the APC gene by a retrotransposal insertion of L1 sequence in a colon cancer. Cancer Res. 1992;52:643-5.

50. Lee E, Iskow R, Yang L, Gokcumen O, Haseley P, Luquette LJ, et al. Landscape of somatic retrotransposition in human cancers. Science. 2012;337:967-71.

51. Beroukhim R, Getz G, Nghiemphu L, Barretina J, Hsueh T, Linhart D, et al. Assessing the significance of chromosomal aberrations in cancer: methodology and application to glioma. Proc Natl Acad Sci. 2007;104:20007-12.

52. Tubio JMC, Li Y, Ju YS, Martincorena I, Cooke SL, Tojo M, et al. Extensive transduction of nonrepetitive DNA mediated by $L 1$ retrotransposition in cancer genomes. Science. 2014:345:1251343.

53. Shukla R, Upton KR, Muñoz-Lopez M, Gerhardt DJ, Fisher ME, Nguyen T, et al. Endogenous retrotransposition activates oncogenic pathways in hepatocellular carcinoma. Cell. 2013;153:101-11.

54. Stetson DB, Ko JS, Heidmann T, Medzhitov R. Trex1 prevents cell-intrinsic initiation of autoimmunity. Cell. 2008;134:587-98.

55. Han BW, Zamore PD. piRNAs. Curr Biol. 2014;24:R730-3.

56. Kim VN, Han J, Siomi MC. Biogenesis of small RNAs in animals. Nat Rev Mol Cell Biol. 2009:10:126-39.

57. Brennecke J, Aravin AA, Stark A, Dus M, Kellis M, Sachidanandam R, et al. Discrete small RNA-generating loci as master regulators of transposon activity in Drosophila. Cell. 2007;128:1089-103.

58. Luteijn MJ, Ketting RF. PIWI-interacting RNAs: from generation to transgenerational epigenetics. Nat Rev Genet. 2013;14:523-34.
59. Aravin AA, Sachidanandam R, Bourc'his D, Schaefer C, Pezic D, Toth KF, et al. A piRNA pathway primed by individual transposons is linked to de novo DNA methylation in mice. Mol Cell. 2008;31:785-99.

60. De Fazio S, Bartonicek N, Di Giacomo M, Abreu-Goodger C, Sankar A, Funaya $C$, et al. The endonuclease activity of Mili fuels piRNA amplification that silences LINE1 elements. Nature. 2011;480:259-63.

61. Gou L-T, Dai P, Yang J-H, Xue Y, Hu Y-P, Zhou Y, et al. Pachytene piRNAs instruct massive mRNA elimination during late spermiogenesis. Cell Res. 2014;24:680-700

62. Shirayama M, Seth M, Lee H-C, Gu W, Ishidate T, Conte D, et al. piRNAs initiate an epigenetic memory of nonself RNA in the $\mathrm{C}$. elegans germline. Cell. 2012;150:65-77.

63. Buckley BA, Burkhart KB, Gu SG, Spracklin G, Kershner A, Fritz H, et al. A nuclear Argonaute promotes multigenerational epigenetic inheritance and germline immortality. Nature. 2012;489:447-51.

64. Ashe A, Sapetschnig A, Weick E-M, Mitchell J, Bagijn MP, Cording AC, et al. piRNAs can trigger a multigenerational epigenetic memory in the germline of C. elegans. Cell. 2012;150:88-99.

65. Conine CC, Moresco JJ, Gu W, Shirayama M, Conte D, Yates JR, et al. Argonautes promote male fertility and provide a paternal memory of germline gene expression in C. elegans. Cell. 2013;155:1532-44.

66. Weick E-M, Miska EA. piRNAs: from biogenesis to function. Development. 2014:141:3458-71.

67. Mochizuki K, Gorovsky MA. Conjugation-specific small RNAs in Tetrahymena have predicted properties of scan (scn) RNAs involved in genome rearrangement. Genes Dev. 2004;18:2068-73.

68. Fang W, Wang $X$, Bracht JR, Nowacki M, Landweber LF. Piwi-interacting RNAs protect DNA against loss during Oxytricha genome rearrangement. Cell. 2012;151:1243-55.

69. Watanabe T, Lin H. Posttranscriptional regulation of gene expression by Piwi proteins and piRNAs. Mol Cell. 2014;56:18-27.

70. Faulkner GJ, Kimura Y, Daub CO, Wani S, Plessy C, Irvine KM, et al. The regulated retrotransposon transcriptome of mammalian cells. Nat Genet. 2009:41:563-71.

71. Rouget C, Papin C, Boureux A, Meunier A-C, Franco B, Robine N, et al. Maternal mRNA deadenylation and decay by the piRNA pathway in the early Drosophila embryo. Nature. 2010;467:1128-32.

72. Watanabe T, Takeda A, Tsukiyama T, Mise K, Okuno T, Sasaki H, et al. Identification and characterization of two novel classes of small RNAs in the mouse germline: retrotransposon-derived siRNAs in oocytes and germline small RNAs in testes. Genes Dev. 2006;20:1732-43.

73. Lin H, Spradling AC. A novel group of pumilio mutations affects the asymmetric division of germline stem cells in the Drosophila ovary. Development. 1997;124:2463-76.

74. Ross RJ, Weiner MM, Lin H. PIWI proteins and PIWI-interacting RNAs in the soma. Nature. 2014;505:353-9.

75. Qiao D, Zeeman A-M, Deng W, Looijenga LHJ, Lin H. Molecular characterization of hiwi, a human member of the piwi gene family whose overexpression is correlated to seminomas. Oncogene. 2002;21:3988-99.

76. Zhao Y-M, Zhou J-M, Wang L-R, He H-W, Wang X-L, Tao Z-H, et al. HIWI is associated with prognosis in patients with hepatocellular carcinoma after curative resection. Cancer. 2012:118:2708-17.

77. Yan H, Wu Q-L, Sun C-Y, Ai L-S, Deng J, Zhang L, et al. piRNA-823 contributes to tumorigenesis by regulating de novo DNA methylation and angiogenesis in multiple myeloma. Leukemia. 2014;29:196-206.

78. Lee JH, Schütte D, Wulf G, Füzesi L, Radzun H-J, Schweyer S, et al. Stem-cell protein Piwil2 is widely expressed in tumors and inhibits apoptosis through activation of Stat3/Bcl-XL pathway. Hum Mol Genet. 2006;15:201-11.

79. Wang $Y$, Liu $Y$, Shen $X$, Zhang $X$, Chen $X$, Yang $C$, et al. The PIWI protein acts as a predictive marker for human gastric cancer. Int J Clin Exp Pathol. 2012:5:315-25

80. Taubert H, Greither T, Kaushal D, Würl P, Bache M, Bartel F, et al. Expression of the stem cell self-renewal gene Hiwi and risk of tumour-related death in patients with soft-tissue sarcoma. Oncogene. 2006;26:1098-100.

81. Grochola LF, Greither T, Taubert H, Möller P, Knippschild U, Udelnow A, et al. The stem cell-associated Hiwi gene in human adenocarcinoma of the pancreas: expression and risk of tumour-related death. $\mathrm{Br} J$ Cancer. 2008;99:1083-8.

82. Sun G, Wang Y, Sun L, Luo H, Liu N, Fu Z, et al. Clinical significance of Hiwi gene expression in gliomas. Brain Res. 2011;1373:183-8. 
83. He W, Wang Z, Wang Q, Fan Q, Shou C, Wang J, et al. Expression of HIWI in human esophageal squamous cell carcinoma is significantly associated with poorer prognosis. BMC Cancer. 2009;9:426

84. He G, Chen L, Ye Y, Xiao Y, Hua K, Jarjoura D, et al. Piwil2 expressed in various stages of cervical neoplasia is a potential complementary marker for p16INK4a. Am J Transl Res. 2010;2:156-69.

85. Zeng Y, Qu L, Meng L, Liu C, Dong B, Xing X, et al. HIWI expression profile in cancer cells and its prognostic value for patients with colorectal cancer. Chin Med J (Engl). 2011;124:2144-9.

86. Liu JJ, Shen R, Chen L, Ye Y, He G, Hua K, et al. Piwil2 is expressed in various stages of breast cancers and has the potential to be used as a novel biomarker. Int J Clin Exp Pathol. 2010;3:328-37.

87. Mei Y, Clark D, Mao L. Novel dimensions of piRNAs in cancer. Cancer Lett. 2013;336:46-52.

88. Cheng J, Deng H, Xiao B, Zhou H, Zhou F, Shen Z, et al. piR-823, a novel non-coding small RNA, demonstrates in vitro and in vivo tumor suppressive activity in human gastric cancer cells. Cancer Lett. 2012;315:12-7.

89. De Cecco M, Criscione SW, Peckham EJ, Hillenmeyer S, Hamm EA, Manivannan J, et al. Genomes of replicatively senescent cells undergo global epigenetic changes leading to gene silencing and activation of transposable elements. Aging Cell. 2013;12:247-56.

90. Van Meter M, Kashyap M, Rezazadeh S, Geneva AJ, Morello TD, Seluanov A, et al. SIRT6 represses LINE1 retrotransposons by ribosylating KAP1 but this repression fails with stress and age. Nat Commun. 2014;5:5011.

91. He L, Thomson JM, Hemann MT, Hernando-Monge E, Mu D, Goodson S, et al. A microRNA polycistron as a potential human oncogene. Nature. 2005;435:828-33

92. Costinean S, Zanesi N, Pekarsky Y, Tili E, Volinia S, Heerema N, et al. Pre-B cell proliferation and lymphoblastic leukemia/high-grade lymphoma in Eu-miR155 transgenic mice. Proc Natl Acad Sci. 2006;103:7024-9.

93. Medina PP, Nolde M, Slack FJ. OncomiR addiction in an in vivo model of microRNA-21-induced pre-B-cell lymphoma. Nature. 2010;467:86-90.

94. Janic A, Mendizabal L, Llamazares S, Rossell D, Gonzalez C. Ectopic expression of germline genes drives malignant brain tumor growth in Drosophila. Science. 2010;330:1824-7.

95. Strome S, Lehmann R. Germ versus soma decisions: lessons from flies and worms. Science. 2007;316:392-3.

96. Curran SP, Wu X, Riedel CG, Ruvkun G. A soma-to-germline transformation in long-lived Caenorhabditis elegans mutants. Nature. 2009;459:1079-84.

97. Simpson AJG, Caballero OL, Jungbluth A, Chen Y-T, Old LJ. Cancer/testis antigens, gametogenesis and cancer. Nat Rev Cancer. 2005;5:615-25.

98. Chi SW, Zang JB, Mele A, Darnell RB. Argonaute HITS-CLIP decodes microRNA-mRNA interaction maps. Nature. 2009;460:479-86.

99. Helwak A, Tollervey D. Mapping the miRNA interactome by cross-linking ligation and sequencing of hybrids (CLASH). Nat Protoc. 2014;9:711-28.

100. Vourekas A, Zheng Q, Alexiou P, Maragkakis M, Kirino Y, Gregory BD, et al. Mili and Miwi target RNA repertoire reveals piRNA biogenesis and function of Miwi in spermiogenesis. Nat Struct Mol Biol. 2012;19:773-81.

\section{Submit your next manuscript to BioMed Central and take full advantage of:}

- Convenient online submission

- Thorough peer review

- No space constraints or color figure charges

- Immediate publication on acceptance

- Inclusion in PubMed, CAS, Scopus and Google Scholar

- Research which is freely available for redistribution

Submit your manuscript at www.biomedcentral.com/submit 